\title{
Dynamic equivalent of an active distribution network taking into account model uncertainties
}

\author{
Gilles Chaspierre \\ Dept. of Elec. Eng. and Comp. Science \\ University of Liège, Belgium \\ g.chaspierre@uliege.be
}

\author{
Guillaume Denis Patrick Panciatici \\ Research \& Development Dept. \\ RTE, Paris La Défense, France \\ patrick.panciatici@rte-france.com
}

\author{
Thierry Van Cutsem \\ Fund for Scientific Research (FNRS) \\ University of Liège, Belgium \\ t.vancutsem@uliege.be
}

\begin{abstract}
A reduced-order model of a distribution network hosting inverter-based generators is derived. This model aims to be used in dynamic simulations of large disturbances in the transmission system. Uncertainties affecting the parameters of the component models are addressed using Monte-Carlo simulations. For a given disturbance, the average and the standard deviation of the randomized system evolutions are extracted. The dynamic equivalent is of the "grey-box" type. Its parameters are adjusted to match as closely as possible the average system response. This involves minimizing a weighted least-square objective function, in which the weights reflect the dispersion of the randomized Monte-Carlo responses. Multiple disturbances are considered to avoid over-fitting, and a recursive procedure is used to select a small subset of them from which the parameters of the ADN equivalent are identified. Simulations results are reported on the CIGRE MV distribution network benchmark. They show a good accuracy of the equivalent.
\end{abstract}

Index Terms-Active distribution networks, dynamic equivalent, inverter-based generators, Monte-Carlo simulations, parameter identification, uncertain dynamic systems.

\section{INTRODUCTION}

A major mutation experienced by power systems is the replacement of conventional generation units feeding the transmission grid by dispersed Inverter-Based Generators (IBGs), such as photo-voltaic units or generators driven by wind turbines, connected at distribution level. The latter play an increasing role in power system control, leading to the concept of Active Distribution Network (ADN).

It becomes important for Transmission System Operators (TSOs) to correctly model those ADNs in their dynamic simulations and stability studies. Maintaining a combined transmission-distribution model is intractable owing to the amount and confidentiality of data handled by Distribution System Operators (DSOs). Instead, it makes sense for the DSOs to process the data of their own systems and transmit to the TSO "anonymized", reduced-order models of their ADNs. The models considered in this paper aim at being incorporated by the TSO in the model of its transmission system used in dynamic studies.

The first step is to set up a model of the unreduced $\mathrm{ADN}$. One issue in this task is the uncertainty affecting the component models. For instance, it is common to resort to an equivalent motor to represent the cumulated effects of a population of motors, but its parameters are usually set to "typical" values (e.g. [1]). As regards IBGs, grid codes specify a range of admissible behaviours, but leave the manufacturers with some freedom in the design of their equipments. A standard approach to deal with such uncertainty consists of performing Monte-Carlo (MC) simulations [2], involving in this case random variations of the model parameters. Thus, for a given disturbance and an initial operating point, a set of randomized time responses is generated [3]. They are processed to derive a reference response, as well as bounds enclosing the latter and indicating how far it is likely to deviate from that reference [4].

The second step is the derivation of a reduced-order model able to render the reference response, from which the deviations should remain acceptable. This dynamic equivalent should offer a good compromise between accuracy and simplicity. The one considered in this paper is of the "grey-box" type as defined and recommended in [5] and [6]. This involves a known, intuitively appealing model whose parameters have to be identified in order to fit the dynamic responses.

The rest of the paper is organized as follows. Section II describes the ADN model and its randomization in MC simulations. Section III details the identification of the ADN equivalent. Simulations results are reported in Section IV. Conclusions are offered Section V.

This paper builds on the authors' previous work reported in [4], [7], [8]. A new objective function taking uncertainty into account, and a new training procedure are proposed for the derivation of the equivalent, while simulation results on a new test system are reported.

\section{ADN MODELLING AND MC SIMULATIONS}

The ADN model includes a standard representation of the network, as well as dynamic models of loads and IBGs.

\section{A. Load model}

The load model is sketched in Fig. 1. The initial load power is split into a static exponential model and an induction motor with third-order model. The motor consumes initially a fraction $f$ of the active power. The compensation capacitor is adjusted to meet a specified power factor $\cos \phi_{m}$.

\section{B. IBG model}

IBGs are represented by a generic model that focuses on interaction with the grid after a disturbance. The values of the 


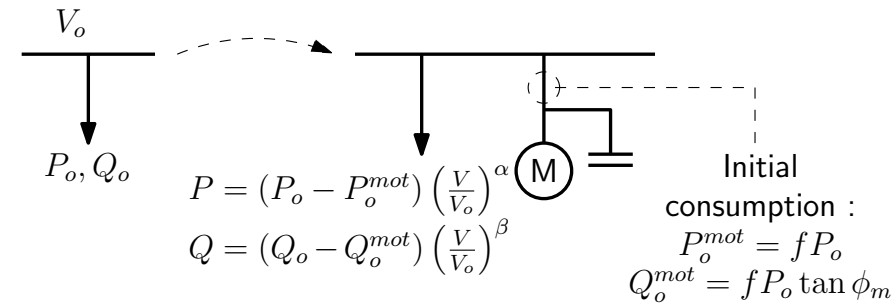

Fig. 1. Load decomposition into exponential and motor parts

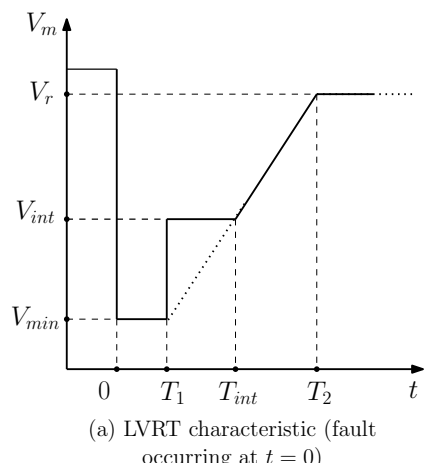

Fig. 2. IBG controls responding to large voltage deviations

parameters were chosen to obey specific grid codes [9]-[11]. The main controls are described hereafter. More details and the complete block diagram can be found in [7], [8].

1) Phase Locked Loop (PLL): The PLL determines the phase angle of the terminal voltage phasor. The magnitude of the current phasor and its phase shift with respect to the voltage phasor are adjusted in order to generate the required active and reactive currents. The PLL dynamics are modeled in some detail. Its response time can be adjusted. A minimum voltage threshold is also specified, under which the PLL is blocked to avoid instability in low voltage situations [12].

2) Low Voltage Ride-Through (LVRT): According to grid codes, IBGs are requested to remain connected to the grid as long as their terminal voltage stays above a reference curve; otherwise, they are allowed to disconnect. The reference curve with six parameters shown in Fig. 2.a has been considered.

3) Reactive current injection: Grid codes require that, if their terminal voltage falls below a threshold value $V_{Q}$, largecapacity IBGs inject reactive current into the grid in order to support the voltage. The injected current varies linearly with the terminal voltage, as shown in Fig. 2.b, where $V_{m}$ is the measured voltage magnitude, $i_{Q o}$ the initial reactive current and $I_{\text {nom }}$ the nominal current of the inverter.

4) Rate of the active current recovery: When an IBG is called to support the grid voltage, it may happen that its active current is reduced to leave room for its reactive counterpart without exceeding the $I_{\text {nom }}$ limit. Once the voltage has recovered to normal values, the IBG active current ramps back to its pre-disturbance value. The recovery cannot be too fast but should not be too slow either, to avoid long-lasting power imbalances. In accordance with some grid codes (e.g. [11]) the rate of recovery of the active current has been chosen in a specified range of values.

\section{Model randomization in MC simulations}

As mentioned in the Introduction, MC simulations are used to assess the impact of model uncertainties. It is assumed that the individual behaviour of each load or IBG is correctly captured by the analytical models presented previously, but the values of the involved parameters are uncertain.

Consider an ADN feeding $n_{L}$ loads and hosting $n_{G}$ IBGs. Let $\pi_{L}$ (resp. $\pi_{G}$ ) be the number of parameters of the load (resp. IBG) model. Thus, the vector $\boldsymbol{p}$ of parameters of the detailed ADN model involves $n_{L} \pi_{L}+n_{G} \pi_{G}$ components. The latter are treated as independent, uniformly distributed random variables with $\boldsymbol{p}^{\text {min }} \leq \boldsymbol{p} \leq \boldsymbol{p}^{\text {max }}$, where $\boldsymbol{p}^{\text {min }}$ and $p^{\max }$ are plausible bounds. For instance, the industrial (resp. residential) loads involve "large" (resp."small") motors with parameters randomized around the values given in [1]. Thus, the parameters are randomized from one $\mathrm{MC}$ simulation to another, but also from one load or IBG to another.

The MC simulations are carried out for $d$ "training" disturbances taking place in the transmission system, as discussed in the next section. The variables of interest are the active and reactive powers entering the ADN. Let $s$ be the number of MC simulations. For the $j$-th disturbance $(j=1, \ldots, d)$ and at a discrete time instant $k$, the following information is extracted from the MC simulations:

$P\left(j, k, \boldsymbol{p}_{i}\right)$ the active power at time $k$ obtained with $\boldsymbol{p}_{i}$, the $i$-th instance of $\boldsymbol{p}(i=1, \ldots, s)$

$Q\left(j, k, \boldsymbol{p}_{i}\right)$ the corresponding reactive power

$\mu_{P}(j, k)$ the average of $P\left(j, k, \boldsymbol{p}_{i}\right)$ over the $s$ instances of $\boldsymbol{p}_{i}$ $\mu_{Q}(j, k)$ the corresponding average for reactive power

$\sigma_{P}(j, k)$ the standard deviation of $P\left(j, k, \boldsymbol{p}_{i}\right)$ over the $s$ instances of $\boldsymbol{p}_{i}$

$\sigma_{Q}(j, k)$ the corresponding standard deviation for reactive power.

The number $s$ of MC simulations is a compromise between the representativity of the randomly drawn sample and the associated computational burden. A procedure for the automatic choice of $s$ was proposed in [4].

\section{IDENTIFICATION OF THE ADN EQUIVALENT}

\section{A. Structure of the equivalent}

The structure of the "grey-box" equivalent is shown in Fig. 3. A distribution grid with a single feeding transformer is considered. The originally distributed IBGs (resp. loads) are aggregated into equivalent IBGs (resp. loads). They can be differentiated according to their location in the grid, technical characteristics and/or voltage level. The aggregated components are placed behind equivalent impedances accounting for the network effects.

The structure shown in Fig. 3 can accommodate various configurations. Three variants are presented in Table I.

In Variant 1 , all loads are aggregated in one equivalent load and all IBGs in one equivalent IBG, both connected to bus 1 


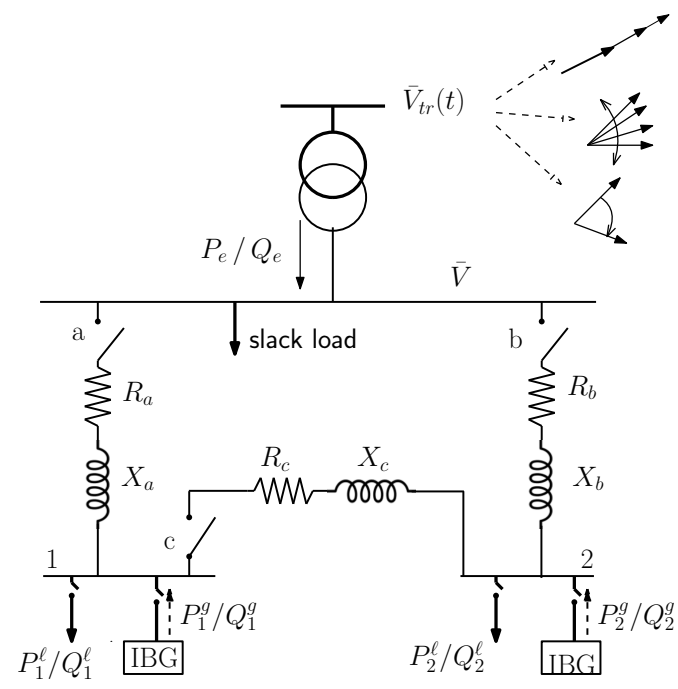

Fig. 3. Structure of the equivalent

TABLE I

EXAMPLE OF STRUCTURES FOR THE EQUIVALENT

\begin{tabular}{|c|c|c|c|}
\hline & VARIANT 1 & VARIANT 2 & VARIANT 3 \\
\hline \hline switch a & closed & closed & open \\
\hline switch b & open & closed & closed \\
\hline switch c & open & open & closed \\
\hline \hline$P_{1}^{\ell} / Q_{1}^{\ell}$ & lumped load & lumped load & load at LV level \\
\hline$P_{1}^{g} / Q_{1}^{g}$ & lumped IBG & - & IBG at LV level \\
\hline$P_{2}^{\ell} / Q_{2}^{\ell}$ & - & - & load at MV level \\
\hline$P_{2}^{g} / Q_{2}^{g}$ & - & lumped IBG & IBG at MV level \\
\hline
\end{tabular}

behind the equivalent impedance $R_{a}+j X_{a}$. Bus 2 and the other two impedances are not used.

Variant 2 is preferred if the loads and IBGs are at very distinct places in the distribution grid. Bus 1, connected through the impedance $R_{a}+j X_{a}$, gathers the aggregated loads while bus 2 , connected through $R_{b}+j X_{b}$, gathers the lumped IBGs. The third impedance is not used.

Variant 3 distinguishes between loads and IBGs connected at Medium Voltage (MV) and Low Voltage (LV) buses, respectively. The former are aggregated at bus 2 , behind the impedance $R_{b}+j X_{b}$ accounting for the MV grid effects, while the latter are lumped at bus 1 , connected to bus 2 through the impedance $R_{c}+j X_{c}$ accounting for the $\mathrm{LV}$ network effects.

\section{B. Load and IBG models in the ADN equivalent}

The lumped load model used in the equivalent is identical to the individual load model in the unreduced system (see Fig. 1).

Similarly, the aggregated IBG model used in the equivalent includes all the features presented in Section II-B. In addition, it accounts for the tripping of a subset of IBGs. Indeed, the individual voltages differ from one IBG bus to another. Hence, it may happen that some IBGs trip and the others remain connected. Since the equivalent IBG has a single terminal voltage, the above situation is accounted for by providing the equivalent IBG with a "partial tripping" feature [7], [8]. The latter consists of multiplying the output current by a factor $f_{1} f_{2} f_{3}$ with $0 \leq f_{1}, f_{2}, f_{3} \leq 1$, where $f_{1}, f_{2}$ and $f_{3}$ relate respectively to the time intervals $\left[0 T_{1}\right],\left[T_{1} T_{\text {int }}\right]$ and $\left[T_{\text {int }} T_{2}\right]$ of the LVRT curve in Fig. 2.a.

\section{Least-square parameter identification}

To avoid over-fitting one particular scenario, the ADN equivalent is identified from time evolutions relative to $d$ different disturbances. The latter are produced by replacing the transmission system with a voltage source $\bar{V}_{t r}(t)$ (see Fig. 3) and imposing large variations of its amplitude, its phase angle or its frequency. Note that the substation transformer is retained explicitly in the equivalent.

Having collected in $\boldsymbol{\theta}$ all parameters to identify, this vector is adjusted so that, at all discrete times $k$ and for all disturbances $j$, the active power $P_{e}(\boldsymbol{\theta}, j, k)$ (resp. reactive power $\left.Q_{e}(\boldsymbol{\theta}, j, k)\right)$ entering the equivalent approaches the average active power $\mu_{P}(j, k)$ (resp. reactive power $\mu_{Q}(j, k)$ ) obtained from MC simulations of the unreduced ADN.

Thus, the following constrained optimization is considered:

$$
\begin{aligned}
\min _{\boldsymbol{\theta}} F(\boldsymbol{\theta})= & \frac{1}{d} \sum_{j=1}^{d}\left[F_{P}(\boldsymbol{\theta}, j)+F_{Q}(\boldsymbol{\theta}, j)\right] \\
\text { with } F_{P}(\boldsymbol{\theta}, j)= & \frac{1}{N} \sum_{k=1}^{N}\left[\frac{P_{e}(\boldsymbol{\theta}, j, k)-\mu_{P}(j, k)}{\sigma_{P}(j, k)}\right]^{2} \\
F_{Q}(\boldsymbol{\theta}, j)= & \frac{1}{N} \sum_{k=1}^{N}\left[\frac{Q_{e}(\boldsymbol{\theta}, j, k)-\mu_{Q}(j, k)}{\sigma_{Q}(j, k)}\right]^{2} \\
& \boldsymbol{\theta}^{L} \leq \boldsymbol{\theta} \leq \boldsymbol{\theta}^{U}
\end{aligned}
$$

where $N$ is the number of discrete times of the simulation. The bounds $\boldsymbol{\theta}^{L}$ and $\boldsymbol{\theta}^{U}$ keep $\boldsymbol{\theta}$ in realistic ranges of values, and are used to generate an initial guess.

Note that each term in the least-square function (2) is weighted by the inverse of the variance $\sigma_{P}^{2}(j, k)$. By so doing, at a given time $k$, the deviation from $\mu_{P}(j, k)$ is penalized more heavily if a small dispersion of the active power values $P\left(j, k, \boldsymbol{p}_{i}\right)(i=1, \ldots, s)$ was observed in the $s$ MC simulations. Conversely, if a large dispersion was observed, denoting a large impact of parameter uncertainty, the deviation of the equivalent from the average behaviour is less penalized. The reactive power terms of the objective function (3) are treated similarly. A minimum value is substituted to $\sigma_{P}$ and/or $\sigma_{Q}$ at times $k$ where the randomized responses are little dispersed.

\section{Initialization}

With reference to Fig. 3, the initial load active and reactive powers $P_{1}^{\ell}, Q_{1}^{\ell}, P_{2}^{\ell}$ and $Q_{2}^{\ell}$ are obtained by summing the corresponding dispersed load powers in the unreduced ADN. Similarly, $P_{1}^{g}, Q_{1}^{g}, P_{2}^{g}$ and $Q_{2}^{g}$ are obtained by summing the corresponding dispersed IBG powers.

Since the equivalent resistances $R_{a}, R_{b}, R_{c}$ and reactances $X_{a}, X_{b}, X_{c}$ are themselves components of the $\boldsymbol{\theta}$ vector, the losses in those impedances change from one value of $\boldsymbol{\theta}$ to another during the optimization. On the other hand, the values of the initial active and reactive powers entering the ADN must not change with $\boldsymbol{\theta}$ (they are forecasted or measured by 
the TSO). Hence, to satisfy the power balance, a "slack" load is added, as shown in Fig. 3. This load is usually small [7].

\section{E. Recursive training procedure}

Clearly, the larger the number $d$ of disturbances, the lower the risk of over-fitting one of them, but the larger the computational burden to identify $\boldsymbol{\theta}$. Hence, a recursive procedure has been devised which consists of adding disturbances progressively in the training set and stopping when the obtained equivalent is found accurate enough with respect to the other, non-trained disturbances. The procedure is as follows.

1. A set of $c$ candidate disturbances is defined. For the $j$-th one $(j=1, \ldots, c)$, MC simulations are performed and the time-varying statistics $\mu_{P}(j, k), \mu_{Q}(j, k), \sigma_{P}(j, k)$ and $\sigma_{Q}(j, k)(k=1, \ldots, N)$ are collected.

2. A small subset of disturbances is selected for initial training $\left(d:=d_{o}\right)$.

3. $\hat{\boldsymbol{\theta}}$ is obtained as the solution of (1-4)..

4. With that value $\hat{\boldsymbol{\theta}}$, the score function $F_{P}(\hat{\boldsymbol{\theta}}, i)+F_{Q}(\hat{\boldsymbol{\theta}}, i)$ is computed for each non-trained disturbance $i$.

5. The worst score is compared to the current value of the objective function (1), i.e.

$$
F_{W S}=\max _{i \in \mathcal{N}}\left[F_{P}(\hat{\boldsymbol{\theta}}, i)+F_{Q}(\hat{\boldsymbol{\theta}}, i)\right] \stackrel{?}{<} F(\hat{\boldsymbol{\theta}})
$$

where $\mathcal{N}$ denotes the set of non-trained disturbances. Note that $F(\hat{\boldsymbol{\theta}})$ is the average score of the $d$ already trained disturbances. If the above test is passed, the training is completed; else, the disturbance with the worst score is added to the training set $(d:=d+1)$. Steps 3 to 5 are repeated, using the last available value $\hat{\boldsymbol{\theta}}$ as initial guess in the optimization.

\section{Simulation RESUlts}

\section{A. Test system and disturbances}

The simulations were performed on the CIGRE MV Distribution Network Benchmark [13], operating in a radial configuration. Its one-line diagram is shown in Fig. 4.

The grid feeds residential as well as industrial loads. It hosts dispersed large capacity and small residential IBGs. The residential IBGs and loads are aggregated models connected behind an equivalent LV impedance as shown in Fig.4.

Due to space limitations, this paper focuses on voltage dips as shown in Fig. 5, obtained by varying $\bar{V}_{t r}$ in Fig. 3. The $c=14$ candidate training disturbances are given in Fig. 5 . $\Delta T=0.10 \mathrm{~s}$ (resp. $0.25 \mathrm{~s}$ ) corresponds to faults in the transmission grid cleared by primary (resp. back-up) protections.

The dynamic evolutions of the active power $P$ and reactive power $Q$ entering the ADN (see Fig. 4) are collected over three seconds. The RAMSES software for dynamic simulation in phasor mode [14] was used; the average time step is $0.01 \mathrm{~s}$.

\section{B. Monte-Carlo simulations}

Using the self-stopping procedure in [4], $s=447$ instances of the parameter vector $\boldsymbol{p}$ of the ADN model were generated, and for each of them, all 14 disturbances were simulated.

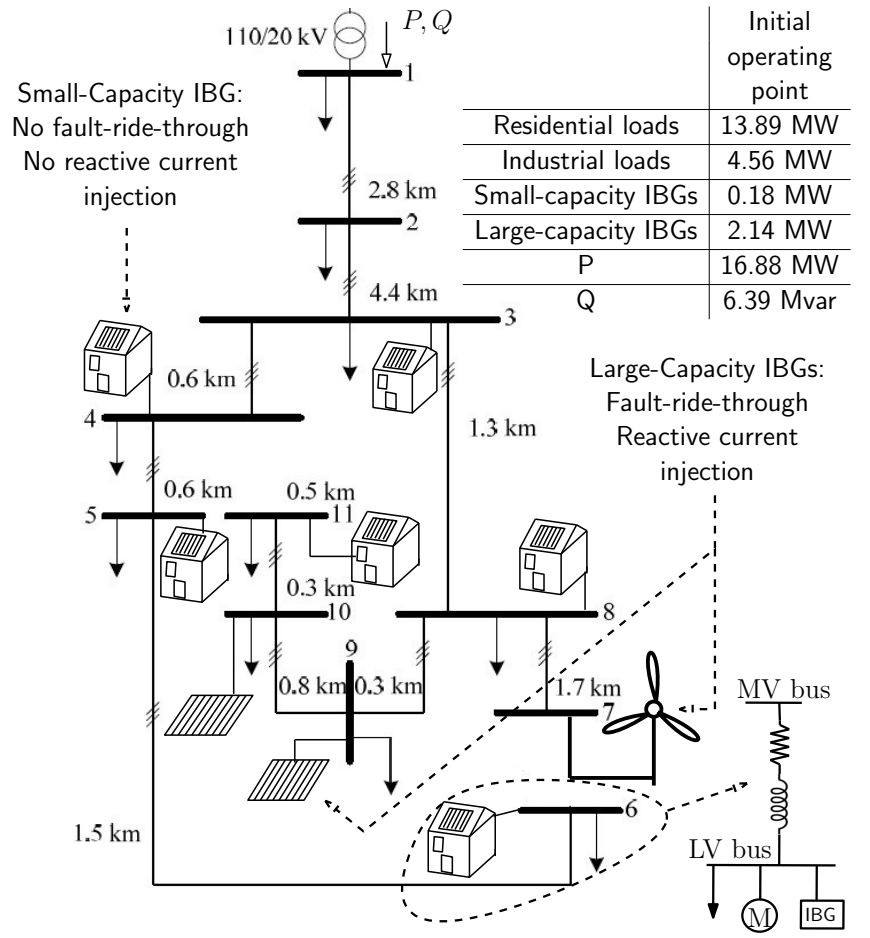

Fig. 4. One-line diagram of CIGRE Medium-Voltage Distribution Network

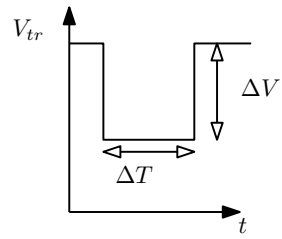

\begin{tabular}{|c|c|c|}
\hline disturb. No & $\Delta V(\mathrm{pu})$ & $\Delta T(\mathrm{~s})$ \\
\hline \hline 1 & 0.2 & 0.10 \\
\hline 2 & 0.2 & 0.25 \\
\hline 3 & 0.3 & 0.10 \\
\hline 4 & 0.3 & 0.25 \\
\hline 5 & 0.4 & 0.10 \\
\hline 6 & 0.4 & 0.25 \\
\hline 7 & 0.5 & 0.10 \\
\hline 8 & 0.5 & 0.25 \\
\hline 9 & 0.6 & 0.10 \\
\hline 10 & 0.6 & 0.25 \\
\hline 11 & 0.7 & 0.10 \\
\hline 12 & 0.7 & 0.25 \\
\hline 13 & 0.8 & 0.1 \\
\hline 14 & 0.8 & 0.25 \\
\hline
\end{tabular}

Fig. 5. Initial set of training disturbances

The following parameters were randomized:

- motor part of each load: three inductances, stator and rotor resistances, inertia constant, fraction of quadratic mechanical torque, load factor [1], fraction $f$ and initial power factor $\cos \phi_{m}$ (see Fig. 1);

- static part of each load: exponents $\alpha$ and $\beta$ (see Fig. 1)

- each IBG: rate of active current recovery, equivalent time constant of current controllers, time constant of PLL and voltage below which it is blocked, $k_{R C I}$ slope

Figures 6 and 7 show the 447 time evolutions of respectively $P$ and $Q$, in response to disturbance No 10. All curves start from the same value since a single operating point is considered here. The overall evolution is explained as follows. During the voltage dip the load with exponential model decreases, while the large-capacity IBGs sacrifice active power to inject reactive power. The load with exponential model recovers together with $V_{t r}$. Some additional power is drawn 


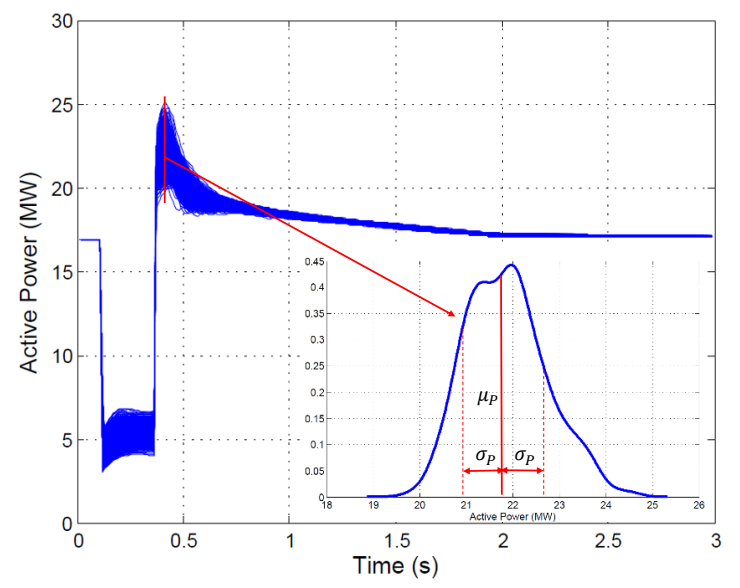

Fig. 6. Randomized evolutions of $P$ for disturbance No 10

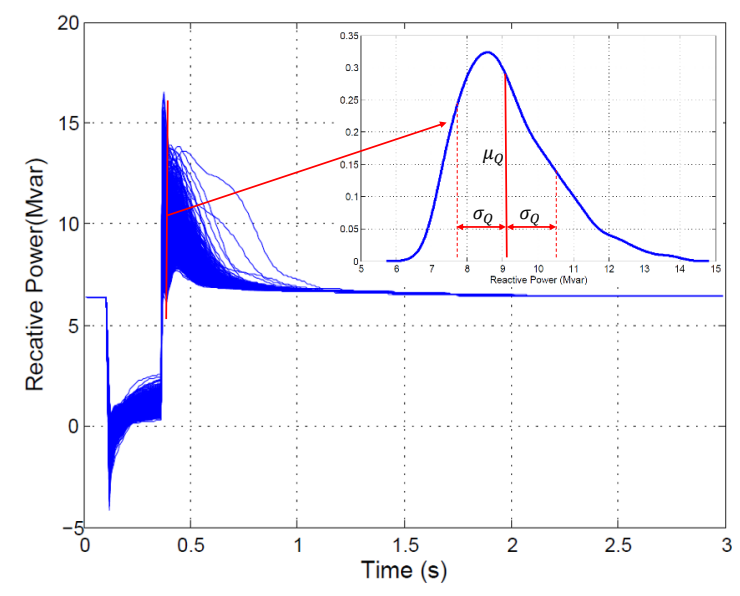

Fig. 7. Randomized evolutions of $Q$ for disturbance No 10

transiently by the re-accelerating motors. The large-capacity IBGs return to unit power factor while ramping up their active power. $P$ settles slightly above its value before disturbance, due to the undervoltage tripping of the small-capacity IBGs.

The figures also show the distributions of the 447 power values at one time after the voltage dip. They are not far from Gaussian distributions. The averages $\mu_{P}(j, k)$ and $\mu_{Q}(j, k)$, and the standard deviations $\sigma_{P}(j, k)$ and $\sigma_{Q}(j, k)$ are extracted at all times $k$, as explained in Section II-C.

\section{Structure of the equivalent}

Owing to the different behaviours of large- and smallcapacity IBGs, the structure chosen for the equivalent corresponds to Variant 3 in Table I, with $R_{b}+j X_{b}$ accounting for the MV grid, and $R_{c}+j X_{c}$ for the LV networks. The aggregated load and IBG attached to bus 1 account for residential loads and small-capacity IBGs while those attached to bus 2 relate to industrial loads and large-capacity IBGs.

\section{Solving the optimization problem (1-4)}

The $\boldsymbol{\theta}$ vector has 40 components to identify.

Since an analytical expression of - at least - the gradient of the objective function (1) with respect to $\theta$ cannot be derived, standard mathematical programming methods can hardly be

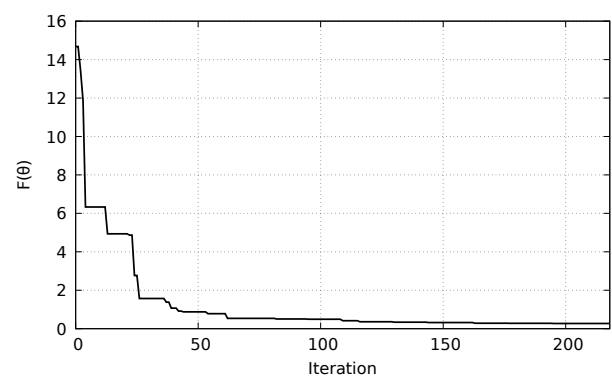

Fig. 8. Example of decrease of the objective function (1) with the number of iterations of the DE algorithm

TABLE II

RESULTS OF RECURSIVE TRAINING

\begin{tabular}{|c|c|c|c|c|}
\hline $\begin{array}{c}\text { Training } \\
\text { iteration }\end{array}$ & $\begin{array}{c}\text { Trained } \\
\text { disturbances }\end{array}$ & $F(\hat{\boldsymbol{\theta}})$ & $F_{W S}$ & $\begin{array}{c}\text { Nb of DE } \\
\text { iterations }\end{array}$ \\
\hline \hline 1 & 7,8 & 0.749 & 291.3 & 100 \\
\hline 2 & $7,8,3$ & 0.2546 & 2.86 & 189 \\
\hline 3 & $7,8,3,5$ & 0.272 & 0.248 & 218 \\
\hline
\end{tabular}

envisaged to solve the above least-square problem. Therefore a derivative-free, metaheuristic optimization method has been chosen, namely a Differential Evolution (DE) algorithm [15]. The open source Python code made available in [16] has been used. The version of the algorithm used is named $\mathrm{rand} / \mathrm{l} / \mathrm{bin}$ because the vectors $\boldsymbol{\theta}$ are randomly chosen using only one vector difference. The binomial crossover strategy was used to mix the information of the trial and the target vectors. More information on the implementation can be found in [16].

An example of decrease of the objective function (1) with the number of iterations of the DE algorithm is given in Fig. 8. In our tests, the iterations were stopped when, for 20 successive iterations, the objective function had decreased by less than 0.001 between two successive iterations.

\section{E. Recursive training}

Table II shows successive results of the recursive training procedure of Section III-E. The number of iterations of the DE algorithm is also provided.

The procedure starts with $d=d_{o}=2$. Disturbances No 7 and 8 were chosen, for they involve an intermediate voltage drop, and different dip durations. The weighted leastsquare procedure yields an average score $F(\hat{\boldsymbol{\theta}})=0.749$. The $\mathrm{ADN}$ response is checked for the remaining 12 disturbances. The worst score found is $F_{W S}=291.3$, corresponding to disturbance No 3 . Hence, at the second iteration, the latter is added to the training set and a new estimate $\hat{\boldsymbol{\theta}}$ is determined. After three iterations the stopping criterion (5) was satisfied. $d=4$ disturbances were eventually used in the identification.

Figure 9 relates to the active power $P_{e}$ after disturbance No 3. The curves drawn with thin lines were derived from MC simulations. They show the average $\mu_{P}(3, k)(k=1, \ldots, N)$ and the bounds $\mu_{P}(3, k) \pm 3 \sigma_{P}(3, k)$ of the confidence interval obtained from the randomized simulations of the detailed ADN model. The curve with heavy dashed line is the active power rendered by the equivalent for the intermediate $\hat{\boldsymbol{\theta}}$ obtained after 


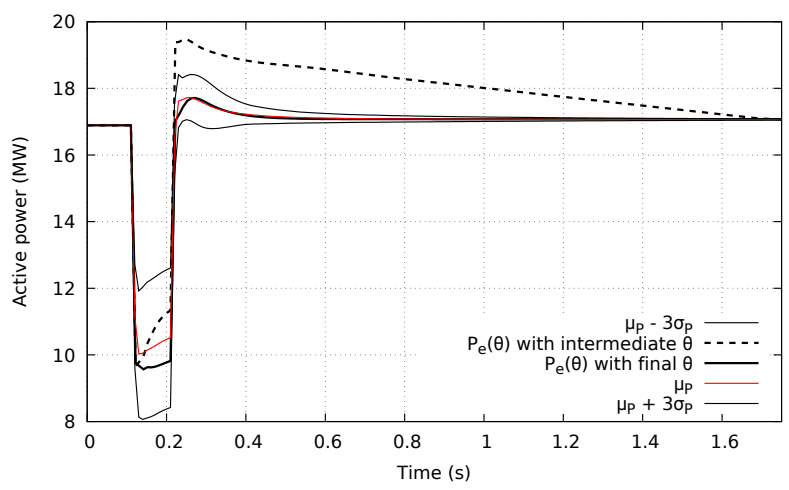

Fig. 9. Active power $P_{e}$ after disturbance No 3: evolution of $\mu_{P}, \mu_{P} \pm 3 \sigma_{P}$, and $P_{e}(\hat{\boldsymbol{\theta}})$ for an intermediate and the final value of $\hat{\boldsymbol{\theta}}$

the first training iteration: see first row in Table II. The plot explains the bad score of that not yet trained disturbance: the rate of active current recovery was badly estimated from the trained disturbances, causing the active power to fall outside the confidence interval for a long duration. The heavy solid curve is the active power given by the equivalent for the final $\hat{\boldsymbol{\theta}}$. Including disturbance No 3 in the training set forced the dynamic response to better approach the average obtained from MC simulations. Similar curves are provided for $Q_{e}$ in Fig. 10.

\section{F. Accuracy of the equivalent}

The accuracy of the equivalent, considering model uncertainty, has been assessed from the maximum absolute "weighted residual" over all $c$ disturbances and all $N$ times:

$$
r_{P}^{\max }=\max _{j=1, \ldots, c} \max _{k=1, \ldots, N}\left|\frac{P_{e}(\hat{\boldsymbol{\theta}}, j, k)-\mu_{P}(j, k)}{\sigma_{P}(j, k)}\right|
$$

The value found is $r_{P}^{\max }=2.05$ during the fault-on period and 1.25 after fault clearing. A similar evaluation for reactive power yields $r_{Q}^{\max }=2.45$ during fault and 1.78 after clearing.

Thus, for both powers the accuracy of the equivalent is very satisfactory, even for non-trained disturbances.

\section{CONClusion}

A methodology has been proposed for identifying an ADN equivalent valid for large disturbances. The model is intended to be used in dynamic simulations of the transmission system.

A weighted least-square objective function is used in the identification, involving statistics extracted from randomized simulations to account for the impact of uncertain parameters in the unreduced model. Furthermore, a recursive training procedure automatically selects a subset of training scenarios from which the parameters of the ADN equivalent are identified.

Simulation results obtained with the CIGRE MV benchmark system show a very satisfactory accuracy of the equivalent. Furthermore, recent results (not reported here) indicate that the equivalent trained with voltage dips covers other types of disturbances, e.g. voltage phase jumps or frequency variations.

The approach is being tested on other systems, so far with the same success.

The impact of the disturbance(s) selected to initialize the recursive training is also being investigated.

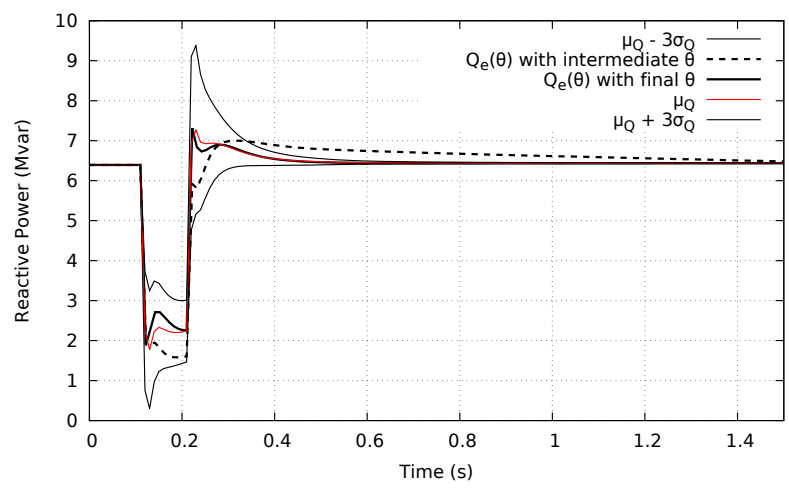

Fig. 10. Reactive power $Q_{e}$ after disturbance No 3: evolution of $\mu_{Q}$, $\mu_{Q} \pm 3 \sigma_{Q}$, and $Q_{e}(\hat{\boldsymbol{\theta}})$ for an intermediate and the final value of $\hat{\boldsymbol{\theta}}$

The next, important step will deal with the training of the equivalent to accommodate a range of operating points.

\section{REFERENCES}

[1] C. Taylor, Power System Voltage Stability. Mc Graw Hill, EPRI Power Engineering Series, 1994.

[2] C. Z. Mooney, Monte Carlo Simulation, vol. 116. Sage Publications, 1997.

[3] I. A. Hiskens, M. Pai, and T. Nguyen, "Bounding uncertainty in power system dynamic simulations," in Power Engineering Society Winter Meeting, 2000. IEEE, vol. 2, pp. 1533-1537, IEEE, 2000.

[4] G. Chaspierre, P. Panciatici, and T. Van Cutsem, "Modelling Active Distribution Networks under Uncertainty: Extracting Parameter Sets from Randomized Dynamic Responses," Proc. 20th PSCC conference, Dublin (Ireland), 2018, in press.

[5] Milanović, Jovica (convener), "Modelling and aggregation of loads in flexible power networks," Report of CIGRE WG C4.605, 2014.

[6] Hatziargyriou, Nikos (convener), "Contribution to bulk system control and stability by distributed energy resources connected at distribution network," IEEE PES Tech. Report PES-TR22, 2017.

[7] G. Chaspierre, P. Panciatici, and T. Van Cutsem, "Aggregated Dynamic Equivalent of a Distribution System hosting Inverter-based Generators," Proc. 20th PSCC conference, Dublin (Ireland), 2018, in press.

[8] G. Chaspierre, P. Panciatici, and T. Van Cutsem, "Dynamic Equivalent of a Distribution Grid Hosting Dispersed Photovoltaic Units," Proc. IREP' 17 Symposium, Espinho (Portugal), 2017, in press.

[9] P. Kotsampopoulos, N. Hatziargyriou, B. Bletterie, and G. Lauss, "Review, analysis and recommendations on recent guidelines for the provision of ancillary services by Distributed Generation," Proc. IEEE Intern. Workshop on Intelligent Energy Systems, pp. 185-190, 2013.

[10] VDE-AR-N 4120, "Technical requirements for the connection and operation of customer installations to the high-voltage network (TCC High-Voltage)," pp. 1-123, January 2015.

[11] B. Weise, "Impact of k-factor and active current reduction during fault-ride-through of generating units connected via voltage-sourced converters on power system stability," IET Renewable Power Generation, vol. 9, no. 1, pp. 25-36, 2015.

[12] B. Weise, "Angle Instability of Generating Units with Fully Rated Converters in Cases of Low Voltages," Chinese Wind and Solar Grid Integration Conference, August 7-8, 2014. Available: $\quad$ http://www.cwpc.cn/cwpp/files/7414/1050/5492/51.Presentation_WiSoGIC2014_Bernd_Weise_Rev2_slides_hidden.pdf.

[13] S. Barsali et al., Benchmark systems for network integration of renewable and distributed energy resources. CIGRE Task Force, 2014.

[14] P. Aristidou, D. Fabozzi, and T. Van Cutsem, "Dynamic simulation of large-scale power systems using a parallel Schur-complement-based decomposition method," IEEE Trans. on Parallel and Distributed Systems, vol. 25, no. 10, pp. 2561-2570, 2014.

[15] K. Price, R. M. Storn, and J. A. Lampinen, Differential Evolution - A Practical Approach to Global Optimization. Springer, 2005.

[16] P. R. Mier, "A tutorial on Differential Evolution with Python," Toulouse (France), September 05, 2017. Available: https://pablormier.github.io/2017/09/05/a-tutorial-on-differentialevolution-with-python/ [Accessed September, 2018]. 